\section{Neurocognitive Dimensions of Breast Cancer and Its Treatment}

Cognitive sequelae of cancer and its treatment have become an active area of investigation (Meyers and Perry, 2008), particularly the effects of adjuvant chemotherapy for breast cancer. Early retrospective neuropsychological studies demonstrated lower function in long-term survivors treated with chemotherapy than in patients not exposed to chemotherapy or healthy controls. These objective data, as well as patient-reported difficulties with cognition in daily life, led to the coining of terms such as 'chemobrain', as alterations in cognitive functioning were presumed to reflect the neurotoxic effects of systemic chemoherapy. Prospective studies later documented lower cognitive performance in a subset of patients prior to adjuvant treatment. These effects remained after controlling for potentially related psychosocial factors (eg, mood, fatigue), suggesting that the cancer disease process may play an independent role. It has also been demonstrated that hormonal therapies may exert an adverse effect on cognition. Reported cognitive effects are generally modest; mean performance for the affected group (eg, chemotherapy-treated $v s$ no chemotherapy, cancer $v s$ noncancer, and hormonal therapy $v s$ no hormonal therapy) typically remains within normal limits based on clinical criteria. Where cognitive changes are noted, the affected domains have been remarkably consistent, with greatest differences noted in processing speed, executive functions such as working memory, and aspects of episodic memory (Jansen et al, 2005).
Psychometric and subjective findings are greatest during and shortly after treatment and resolve in most patients over time, with longitudinal studies showing residual cognitive sequelae in only a subset of patients several years post-treatment. Multiple candidate mechanisms for these effects have been proposed, including chemotherapy-induced DNA damage (directly or through increases in oxidative stress), individual variation in genes related to neural repair and/or plasticity, and/or chemotherapy-induced hormonal changes (Ahles and Saykin, 2007). Structural and functional neuroimaging has been applied to examine the neural substrate of these cognitive changes in both retrospective and prospective samples. Using voxel-based morphometry, a structural MRI technique that is capable of detecting alterations in gray matter density, our group has detected decreases in gray matter in bilateral

TABLE 1 Atlas Coordinates, Cluster Extents, $P$ and $T$ Values, and Region Descriptions

\begin{tabular}{lcccl}
\hline MNI coordinates $(\boldsymbol{x}, \boldsymbol{y}, \boldsymbol{z})$ & Cluster extent $(\boldsymbol{k})$ & Cluster-level $\boldsymbol{P}_{\text {corrected }}$ & $\boldsymbol{T}$ & Region description (for cluster peak) \\
\hline$-44,39,-6$ & 5600 & $<0.001$ & 6.23 & L middle frontal gyrus (BA47) \\
$14,30,54$ & 6063 & $<0.001$ & 5.34 & R superior frontal gyrus (BA8) \\
$52,-2,39$ & 2823 & 0.002 & 5.14 & R precentral gyrus (BA6) \\
$12,56,-16$ & 3895 & $<0.001$ & 4.92 & R superior frontal gyrus (BA11) \\
$-37,-71,-33$ & 4915 & $<0.001$ & 4.74 & L cerebellum \\
$-15,49,36$ & 2734 & 0.003 & 4.45 & $L$ superior frontal gyrus (BA9) \\
$34,6,-45$ & 2568 & 0.004 & 4.35 & R superior temporal gyrus (BA38) \\
$38,-74,-29$ & 1486 & 0.043 & 3.94 & R cerebellum \\
\hline
\end{tabular}

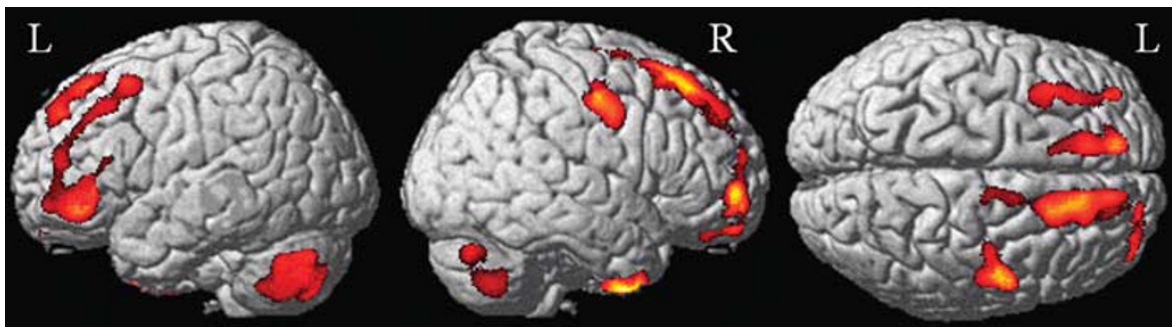

Figure 1. Reduction in brain gray matter density from baseline to 1 month after breast cancer chemotherapy. Data were analyzed using SPM5, with the critical significance threshold $\left(P_{\text {crit }}\right)$ set to 0.001 . Surface renderings show gray matter changes in bilateral frontal, temporal, and cerebellar regions. Reduction in gray matter density was also evident in bilateral medial temporal regions, and in the right thalamus (not shown). 
frontal and temporal regions 1 month after chemotherapy relative to baseline (Figure 1 and Table 1) (McDonald et $a l$, in press). These changes do not occur in breast cancer patients who are not treated with chemotherapy or healthy controls. One year later, these gray matter alterations show partial but not complete recovery, consistent with previous work in retrospective samples. fMRI and PET have also shown differences in brain function during tasks tapping episodic memory and executive functions, including working memory (de Ruiter et al, 2010). Mirroring the cognitive literature, altered patterns of brain activation have been found both prior to adjuvant treatment and following chemotherapy or hormonal treatment. Both structural and functional neuroimaging approaches have shown alteration in frontal brain regions, consistent with the most commonly affected cognitive processes in prior neuropsychological studies. Ongoing research examining variables that contribute to cancer- and treatmentrelated cognitive and brain changes (eg, genetic variability and other bioarkers, age, cognitive reserve, and other medical comorbidities) will be critical to identifying potential risk factors that may increase individual vulnerability (Ahles et al, in press). Preclinical research in animal models can be expected to further enhance the understanding of underlying mechanisms. Finally, identification of optimal treatment approaches will be an important future direction.

\section{ACKNOWLEDGEMENTS}

The work described here was supported in part by National Cancer Institute Grants 5R01CA087845 and 5R01CA101318.

\footnotetext{
Brenna C McDonald ${ }^{\mathbf{1}}$ and Andrew J Saykin $^{1}$

${ }^{1}$ Center for Neuroimaging, Department of Radiology and Imaging Sciences and the Melvin and Bren Simon Cancer Center, Indiana University School of Medicine, Indianapolis, IN, USA

E-mail: asaykin@iupui.edu
}

DISCLOSURE

The authors declare no conflict of interest.

Ahles TA, Saykin AJ (2007). Candidate mechanisms for chemotherapy-induced cognitive changes. Nat Rev Cancer 7: 192-201.

Ahles TA, Saykin AJ, McDonald BC, Li Y, Furstenberg CT, Hanscom BS et al. (2010). Longitudinal assessment of cognitive changes associated with adjuvant treatment for breast cancer: the impact of age and cognitive reserve. J Clin Oncol (in press).

de Ruiter MB, Reneman L, Boogerd W, Veltman DJ, van Dam FSAM, Nederveen AJ et al (2010). Cerebral hyporesponsiveness and cognitive impairment 10 years after chemotherapy for breast cancer. Hum Brain Mapping (in press); e-pub ahead of print 28 July 2010, at http://onlinelibrary. wiley.com/doi/10.1002/hbm.21102/pdf.

Jansen CE, Miaskowski C, Dodd M, Dowling G, Kramer $J$ (2005). A metaanalysis of studies of the effects of cancer chemotherapy on various domains of cognitive function. Cancer 104: 2222-2233.

McDonald BC, Conroy SK, Ahles TA, West JD, Saykin AJ (2010). Gray matter reduction associated with systemic chemotherapy for breast cancer: a prospective MRI study. Breast Cancer Res Treat (in press); e-pub ahead of print 6 August 2010, at http:// www.springerlink.com/content/5686768t1q25287p/ fulltext.pdf.

Meyers CA, Perry JR (eds) (2008). Cognition and Cancer. Cambridge University Press: Cambridge, MA

Neuropsychopharmacology Reviews (2011) 36, 355-356; doi: 10.1038/npp.2010.142

\section{NICO-TEEN: Neural Substrates that Mediate Adolescent Tobacco Abuse}

Adolescents are especially likely to initiate tobacco use and are more vulnerable to long-term tobacco dependence. Although the importance of factors such as environmental conditions, genetics, sex differences, and constituents of tobacco other than nicotine has been recognized, relatively little is known about the neural mechanisms that mediate enhanced sensitivity to tobacco abuse during adolescence.

Recent preclinical studies have led to our working hypothesis that enhanced tobacco abuse during adolescence is promoted by: (1) enhanced positive effects of nicotine; and (2) reduced negative effects of nicotine and withdrawal from this drug during adolescence compared with adulthood
(O'Dell, 2009). Thus, the inadequate balance favoring strong positive effects of nicotine over reduced negative effects produces enhanced vulnerability to tobacco abuse during adolescence.

Much work comparing age differences to nicotine has focused on the mesolimbic dopamine pathway from the ventral tegmental area (VTA) to the nucleus accumbens (NAcc) where dopamine is increased by nicotine but decreased during withdrawal (Mansvelder and McGehee, 2002). These neurochemical effects are age dependent, as nicotine increases NAcc dopamine to a greater extent in adolescent $v s$ adult rats (Shearman et al, 2008). Also, we reported that nicotine withdrawal decreases NAcc dopamine to a lesser extent in adolescent $v s$ adult rats (Natividad et al, 2010). These studies suggest that mesolimbic dopaminergic mechanisms are important in modulating adolescent vulnerability to tobacco abuse.

Our working hypothesis is that the age differences in dopamine have their origin in the VTA where excitatory mechanisms regulate dopamine release in the NAcc. This is based on our observation that nicotine withdrawal decreases glutamate levels in the VTA of adult, but not adolescent, rats. Because excitation in the VTA is not reduced, adolescents show smaller reductions in NAcc dopamine during withdrawal. This hypothesis is consistent with evidence that excitatory systems that facilitate dopamine are overdeveloped during adolescence (McDonald and Johnston, 1990). Taken together, we hypothesize that adolescents show enhanced nicotine reward and reduced withdrawal through enhanced excitation of VTA cell bodies that release dopamine in the NAcc.

Our hypothesis has important clinical implications. First, reduced sensitivity to withdrawal during adolescence implies that the diagnostic criteria developed for tobacco dependence in adults, based primarily on withdrawal, are inappropriate for adolescents. A corollary is that treatments focusing on alleviating withdrawal will 